\title{
Effect of different muscle contraction interventions using an isokinetic dynamometer on muscle recovery following muscle injury
}

\author{
Min-Suk Kang, Jooyoung Kim, Joohyung Lee* \\ Sports, Health and Rehabilitation Major, College of Physical Education, Kookmin University, Seoul, Korea
}

The objective of the present study was to examine the effects of different muscle contraction interventions using isokinetic dynamometers on the muscle recovery after muscle injury caused by eccentric exercise. A total of 28 healthy male adults participated in the present study and each subject was randomly assigned to eccentric muscle contraction $(E C, n=7)$, concentric muscle contraction (CC, $n=7)$, both eccentric and concentric muscle contraction (BEC, $n=7)$, and control (CON, $n=7$ ) groups after performing eccentric exercise. In all groups, except the control group, a different type of muscle contraction intervention was applied repeatedly for 24-96 hr after eccentric exercise, and maximum strength, muscle soreness, and creatine kinase (CK) levels were measured. The results showed a significant association between time and group, with respect to maximum strength $(P<0.05)$, muscle soreness $(P<0.001)$, and CK $(P<0.001)$ after eccentric exercise. Moreover, BEC group showed faster muscle recovery than other groups. In conclusion, implementing active strategies such as performing BEC using an isokinetic dynamometer after eccentric exercise may be effective in facilitating muscle recovery.

Keywords: Concentric, Eccentric, Isokinetic, Muscle injury, Recovery

\section{INTRODUCTION}

Skeletal muscle injuries are common in sports (Järvinen et al., 2014). Several interventions have been used to treat symptoms including muscle soreness, tenderness, and decreased range of motion after muscle injury (Dupuy et al., 2018; Sousa et al., 2014). It has been well documented that muscle injury depends on the type of contraction loaded on the skeletal muscle. Eccentric muscle contractions, in general, induce more injury than concentric or isometric contractions because of excess tension during contractions (Lavender and Nosaka, 2006; Sayers et al., 1999). Such excess tension induces disruption in sarcomeres as well as cell membranes leading to loss of excitation-contraction coupling (Proske and Morgan, 2001).

According to some studies, additional exercise performing in the early recovery phase after eccentric exercise does not exacerbate muscle injury or delay recovery (Chen and Hsieh, 2001; Nosaka and Newton, 2002; Sorichter et al., 1995). Sorichter et al. (1995) reported that light concentric exercise after eccentric exercise does not increase muscle injury, breakdown of connective tissue, or inflammation, and similar results were found in a study by Chen and Hsieh (2001).

Other studies have even reported that additional exercise actually rather decreased the indicators that reflect muscle injury (Donnelly et al., 1992; Saxton and Donnelly, 1995). Saxton and Donnelly (1995) reported that light concentric exercise during the recovery period after eccentric exercise reduced creatine kinase (CK), temporarily relieved muscle soreness, and facilitated recovery of maximum voluntary force, although the effect was minimal. Meanwhile, Donnelly et al. (1992) reported that light eccentric exercise after eccentric exercise had no effect on strength and muscle soreness, but was able to reduce $\mathrm{CK}$.

Such a phenomenon is commonly known as the "repeated bout effect," which refers to the "adaptation effect" of muscles to eccen-

\footnotetext{
${ }^{*}$ Corresponding author: Joohyung Lee (D) https://orcid.org/0000-0001-8279-3830 Sport, Health and Rehabilitation Major, College of Physical Education, Kookmin University, 77 Jeongneung-ro, Seongbuk-gu, Seoul 02707, Korea Tel: +82-2-910-4782, Fax: +82-2-910-4789, E-mail: jolee@kookmin.ac.kr Received: September 6, 2018 / Accepted: November 12, 2018
}

This is an Open Access article distributed under the terms of the Creative Commons Attribution Non-Commercial License (http://creativecommons.org/licenses/by-nc/4.0/) which permits unrestricted non-commercial use, distribution, and reproduction in any medium, provided the original work is properly cited. 
tric exercise (Howatson et al., 2007; Hyldahl et al., 2017; McHugh, 2003). However, most of the previous studies have reported application of only a single type of muscle contraction (concentric or eccentric), whereas there are only limited number of studies that applied both concentric and eccentric muscle contraction (EC) simultaneously. This limitation does not clarify which type of muscle contraction is better for muscle injury caused by eccentric exercise in practical situations.

Therefore, the purpose of this study was to investigate whether three types of muscle contraction interventions including eccentric, concentric, and both, affect muscle recovery following eccentric exercise-induced muscle injury.

\section{MATERIALS AND METHODS}

\section{Subjects}

The subjects of this study were 28 healthy male adults who were working in the National Fitness Center without any comorbidities, any joint dysfunction history, pain, and exercise restriction in the lower body. Each subject was fully explained the purpose of the study and the experimental procedure prior to signing an informed consent form. The purpose of this study and the experimental procedure were fully explained. During the study period, subjects were asked to abstain from any excess physical activity, alcohol consumption, and drug use. Each subject was randomly assigned to either EC, concentric muscle contraction (CC), both eccentric and concentric muscle contraction (BEC), or control $(\mathrm{CON})$ group following eccentric exercise. Characteristics of subjects have been shown in Table 1 .

\section{Eccentric exercise protocol}

Using the isokinetic dynamometer (Biodex System 3 Pro, Biodex Medical Systems, Inc., New York, NY, USA), initially the peak muscle torque was measured in the nondominant leg at an angular velocity of $60^{\circ} / \mathrm{sec}$ thrice. To induce muscle injury, the subjects performed 10 sets of 15 repetitions of maximal ECs at an angular

Table 1. Characteristics of subjects

\begin{tabular}{lcccc}
\hline Characteristic & EC $(n=7)$ & $C C(n=7)$ & $\operatorname{BEC}(n=7)$ & $\operatorname{CON}(n=7)$ \\
\hline Age $(\mathrm{yr})$ & $30.57 \pm 4.15$ & $30.43 \pm 2.82$ & $27.86 \pm 3.13$ & $28.29 \pm 2.56$ \\
Height $(\mathrm{cm})$ & $176.57 \pm 4.99$ & $175.86 \pm 4.52$ & $176.14 \pm 5.32$ & $178.14 \pm 6.03$ \\
Weight $(\mathrm{kg})$ & $77.42 \pm 12.55$ & $76.84 \pm 12.60$ & $76.17 \pm 13.47$ & $80.54 \pm 13.44$
\end{tabular}

Values are presented as mean \pm standard deviation.

$\mathrm{EC}$, eccentric muscle contraction; $\mathrm{CC}$, concentric muscle contraction; $\mathrm{BEC}$, both eccentric and concentric muscle contraction; $\mathrm{CON}$, control.

No statistically significant difference between groups. velocity of $60 \% \mathrm{sec}$ (Lund et al., 1998). The rest time between each set was set to 60 sec.

\section{Muscle contraction intervention}

In each group, a different type of muscle contraction intervention was applied repeatedly for 24-96 hr after eccentric exercise. Only the contraction type differed between the groups, and for all groups, the exercise intensity was set to $30 \%-40 \%$ of the maximum peak torque measured prior to the eccentric exercise. The angular velocity, repetition, and rest time between sets were same as the eccentric exercise protocol and a total of three sets were performed.

\section{Maximum strength}

An isokinetic dynamometer was used to measure the maximum strength of the subjects. The peak torque values of the extensor and flexor muscles in the left and right knee joints were measured and presented as the relative strength. After seating the subjects on the equipment, belts connected to the equipment were used to immobilize the trunk and abdomen, as well as the leg being tested. In addition, shin pads were placed $2 \mathrm{~cm}$ above the ankle and fastened with Velcro straps. To become acclimated to the test, the subjects performed 2-3 repetitions of practice motions, after which the actual measurements began, with the subjects firmly gripping the handle on both sides. During the test, the researcher encouraged the subjects to promote exertion of maximum strength. The angular velocity was set to $60 \% \mathrm{sec}$ and the measurements were repeated 3 times.

\section{Muscle soreness}

The visual analogue scale (VAS) was used to measure muscle soreness. A 100-mm line was used, where the 0-mm mark on the left side indicated no pain with points closer to the 100-mm mark on the right side indicating more severe pain. The subjects personally drew a vertical line on the VAS to indicate the level of pain.

\section{Creatine kinase}

For blood CK measurement, $5 \mathrm{~mL}$ of blood sample was collected from the brachial vein. Blood collection took place in the morning, after the subjects had fasted for at least $8 \mathrm{hr}$. The collected blood samples were centrifuged at 2,500-3,000 rpm for $10-15 \mathrm{~min}$ to separate the serum. Subsequently, a clinical chemistry analyzer (Elitech Selectra XL, Diamond Diagnostics Inc., Massachusetts, MA, USA) was used to measure CK. 
Table 2. Change of maximum strength following muscle injury

\begin{tabular}{|c|c|c|c|c|c|c|c|}
\hline Variable & Pre & Post & $24 \mathrm{hr}$ & $48 \mathrm{hr}$ & $72 \mathrm{hr}$ & $96 \mathrm{hr}$ & $P$-value \\
\hline $\mathrm{EC}(\mathrm{n}=7)$ & $100 \pm 0$ & $49.51 \pm 12.55$ & $69.84 \pm 8.65$ & $67.47 \pm 8.64$ & $73.75 \pm 10.94$ & $78.54 \pm 9.92$ & 0.032 \\
\hline$C C(n=7)$ & $100 \pm 0$ & $55.05 \pm 9.79$ & $78.18 \pm 6.92$ & $68.90 \pm 7.46$ & $82.76 \pm 9.97$ & $89.76 \pm 5.73$ & \\
\hline $\operatorname{BEC}(n=7)$ & $100 \pm 0$ & $63.61 \pm 17.52$ & $76.62 \pm 6.27$ & $87.86 \pm 5.45^{a, b)}$ & $87.57 \pm 11.28$ & $93.21 \pm 10.40$ & \\
\hline $\operatorname{CON}(n=7)$ & $100 \pm 0$ & $65.84 \pm 15.57$ & $74.48 \pm 8.74$ & $75.83 \pm 10.55$ & $80.03 \pm 6.73$ & $88.13 \pm 7.88$ & \\
\hline
\end{tabular}

Values are presented as mean \pm standard deviation (\%).

EC, eccentric muscle contraction; CC, concentric muscle contraction; BEC, both eccentric and concentric muscle contraction; CON, control.

${ }^{a} \mathrm{BEC}$ showed greater maximum strength at $48 \mathrm{hr}$ after eccentric exercise compared to EC $(P<0.01)$. ${ }^{b} \mathrm{BEC}$ showed greater maximum strength at $48 \mathrm{hr}$ after eccentric exercise compared to CC $(P<0.001)$.

Table 3. Change of muscle soreness following muscle injury

\begin{tabular}{|c|c|c|c|c|c|c|c|}
\hline Variable & Pre & Post & $24 \mathrm{hr}$ & $48 \mathrm{hr}$ & $72 \mathrm{hr}$ & $96 \mathrm{hr}$ & $P$-value \\
\hline $\mathrm{EC}(\mathrm{n}=7)$ & $0 \pm 0$ & $5.43 \pm 1.71$ & $7.43 \pm 1.13$ & $8.29 \pm 1.79$ & $6.00 \pm 2.76$ & $3.43 \pm 1.71$ & 0.000 \\
\hline$C C(n=7)$ & $0 \pm 0$ & $4.14 \pm 2.03$ & $5.00 \pm 1.00^{\mathrm{a}}$ & $5.14 \pm 1.77^{c)}$ & $2.71 \pm 0.75$ & $1.71 \pm 0.75$ & \\
\hline $\operatorname{BEC}(n=7)$ & $0 \pm 0$ & $2.71 \pm 1.60$ & $3.86 \pm 1.34^{b)}$ & $4.00 \pm 1.41^{d)}$ & $2.29 \pm 1.11$ & $2.00 \pm 1.82$ & \\
\hline $\operatorname{CON}(n=7)$ & $0 \pm 0$ & $4.14 \pm 1.77$ & $5.86 \pm 1.21$ & $4.14 \pm 1.06$ & $3.14 \pm 1.06$ & $2.43 \pm 0.53$ & \\
\hline
\end{tabular}

Values are presented as mean \pm standard deviation (mm).

EC, eccentric muscle contraction; CC, concentric muscle contraction; BEC, both eccentric and concentric muscle contraction; CON, control.

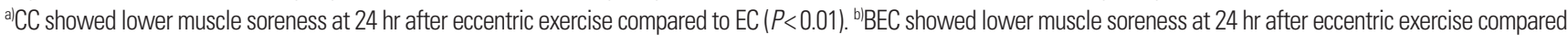
to EC $(P<0.001)$. ${ }^{c} \mathrm{CC}$ showed lower muscle soreness at $48 \mathrm{hr}$ after eccentric exercise compared to EC $(P<0.05)$. ${ }^{d} \mathrm{BEC}$ showed lower muscle soreness at $48 \mathrm{hr}$ after eccentric exercise compared to $\mathrm{EC}(P<0.01)$.

Table 4. Change of creatine kinase (CK) following muscle injury

\begin{tabular}{|c|c|c|c|c|c|c|c|}
\hline Variable & Pre & Post & $24 \mathrm{hr}$ & $48 \mathrm{hr}$ & $72 \mathrm{hr}$ & $96 \mathrm{hr}$ & $P$-value \\
\hline$E C(n=7)$ & $246.14 \pm 155.67$ & $312.00 \pm 169.72$ & $569.43 \pm 270.98$ & $3,218.00 \pm 2,241.96$ & $9,903.43 \pm 5,438.90$ & $19,912.29 \pm 23,294.94$ & 0.001 \\
\hline$C C(n=7)$ & $194.29 \pm 131.38$ & $237.00 \pm 154.36$ & $313.00 \pm 204.77$ & $715.71 \pm 344.55$ & $1,900.57 \pm 597.90^{\mathrm{a})}$ & $4,035.57 \pm 2,797.88$ & \\
\hline $\operatorname{BEC}(n=7)$ & $275.71 \pm 176.21$ & $235.71 \pm 145.39$ & $572.00 \pm 531.71$ & $1,669.14 \pm 1,529.82$ & $3,256.29 \pm 1,930.62$ & $6,297.86 \pm 4,255.48$ & \\
\hline $\operatorname{CON}(n=7)$ & $167.00 \pm 101.89$ & $181.14 \pm 110.61$ & $241.29 \pm 105.59$ & $567.29 \pm 248.49$ & $1,947.00 \pm 667.97^{\text {b) }}$ & $2,476.71 \pm 1,070.94$ & \\
\hline
\end{tabular}

Values are presented as mean \pm standard deviation (U/L).

EC, eccentric muscle contraction; CC, concentric muscle contraction; BEC, both eccentric and concentric muscle contraction; CON, control.

${ }^{a} \mathrm{CC}$ showed lower $\mathrm{CK}$ at $72 \mathrm{hr}$ after eccentric exercise compared to $\mathrm{EC}(P<0.05) .{ }^{\mathrm{b}} \mathrm{CON}$ showed lower $\mathrm{CK}$ at $72 \mathrm{hr}$ after eccentric exercise compared to $\mathrm{EC}(P<0.05)$.

\section{Statistical analysis}

The results were analyzed using IBM SPSS ver. 18.0 (IBM Co., Armonk, NY, USA). All variables were derived as mean and standard deviation. A repeated-measures analysis of variance was used to test the interaction between time and group. The cutoff for statistical significance was set to 0.05 .

\section{RESULTS}

The results in the present study indicated that maximum strength after eccentric exercise showed a significant association with time and group $(P<0.05)$ (Table 2$)$. In all groups, maximum strength decreased significantly immediately after eccentric exercise $(P<$ $0.001)$, but after $48 \mathrm{hr}$, the BEC group showed faster recovery of maximum strength than the other groups $(P<0.01)$.

Muscle soreness and CK also showed a significant association with time and group $(P<0.001)$ (Tables 3, 4). Muscle soreness increased after exercise in all groups, but the BEC and CC groups showed significant decrease as compared to the EC group at 24 (BEC, $P<0.001$; CC, $P<0.01)$ and $48 \mathrm{hr}(\mathrm{BEC}, P<0.01$; CC, $P<$ $0.05)$. However, there was no significant differences between $\mathrm{BEC}$ and $\mathrm{CON}(P>0.05)$. Lastly, $\mathrm{CK}$ showed a significant increase after eccentric exercise in all groups $(P<0.001)$, with the EMC group showing especially high increase as compared to other groups. After $72 \mathrm{hr}$, the CMC group showed a significant decrease in CK as compared to the EMC group $(P<0.05)$.

\section{DISCUSSION}

The purpose of the present study was to examine the effects of different muscle contraction interventions after muscle injury on muscle recovery. The results showed that applying BEC after mus- 
cle injury had a more positive effect on muscle recovery than other muscle contraction. Some studies have reported that EC after muscle injury does not exacerbate muscle injury (Chen and Hsieh, 2001; Nosaka and Newton, 2002), but in the present study, the EC group showed slower muscle recovery than the other groups. In particular, when compared with other groups, it was confirmed that CK in the EC group continued to increase and muscle injury did not improve during the recovery period.

Such results that contradict previous study results may be attributed to each study using different muscles (elbow flexor vs. knee flexor), initial eccentric exercise protocols (especially in intensity), and muscle contraction intervention methods after eccentric exercise. In this study, the intensity of muscle contraction intervention after maximal eccentric exercise of the knee flexor was set to $30 \%-40 \%$ of the maximum peak torque. However, in previous studies that used elbow flexor or chest muscle, the intensity was similar to that of the maximal eccentric exercise conducted earlier (Chen and Hsieh, 2001; Nosaka and Newton, 2002; Smith et al., 1994). Other previous studies differed from the present study, with the exception of the study by Chen and Hsieh (2001), in that muscle contraction intervention was performed only once after the first eccentric exercise (Smith et al., 1994) or nonrepeatedly (Nosaka and Newton, 2002). Chen and Hsieh (2001) performed repeatedly eccentric exercise for 6 days after muscle injury, but Smith et al. (1994) performed eccentric exercise only 2 days after muscle injury, and Nosaka and Newton (2002) performed eccentric exercise just 2 and 4 days after muscle injury.

A noteworthy point in the present study is that, unlike previous studies that applied only a single muscle contraction (Chen and Hsieh, 2001; Donnelly et al., 1992; Saxton and Donnelly, 1995; Sorichter et al., 1995), the present study demonstrated that BEC had a positive effect on recovery. Although the rationale behind such results is not yet clear, BEC, could lead to an increase in the blood flow. Such a change can expedite the removal of metabolic waste and pain-inducing substrates (Cheung et al., 2003; Dupuy et al., 2018). Moreover, "exercise-induced analgesia" may be another reason for reduced muscle soreness. This refers to increased pain threshold and pain tolerance after exercise (Koltyn, 2000). Armstrong (1984) also reported that exercising sore muscles is the most effective way of reducing soreness. In fact, Zainuddin et al. (2006) reported that light exercise performed after eccentric exercise showed temporarily analgesic effect.

However, despite the fact that the recovery of maximal strength was fast in the BEC group, no significant difference was found in terms of the change in CK level. Brancaccio et al. (2007) stated that $\mathrm{CK}$, a blood muscle protein, had no positive correlation with functional markers such as maximal isometric force or range of motion. Other studies also stated that CK levels did not always align with the magnitude of the muscle injury (Manfredi et al., 1991; Margaritis et al., 1999). Margaritis et al. (1999) reported that upon analyzing the change in the muscle injury marker after a triathlon, the maximal isometric strength recovered well to the normal state after 4 days, while the CK level did not decrease but continued to increase. Such results show that CK cannot fully reflect structural damage and recovery of muscle cells. Additional studies are necessary regarding this matter.

In summarizing the results of this study along with those of previous studies, both concentric and eccentric exercises could be a better choice in terms of the muscle contraction intervention applied after eccentric exercise-induced muscle injury in practical situations. In addition, we recommend performing the both concentric and eccentric exercises at a lower intensity than that of the first eccentric exercise and performing repeatedly both concentric and eccentric exercises.

However, the present study has a few limitations. First, the study population did not consist of athletes or untrained individuals. Training status is one of the latent factors that can affect recovery after eccentric exercise (Brancaccio et al., 2007; Kim and Lee, 2015). Therefore, future studies should consider this fact and should examine changes in people who are already well trained. Second, both concentric and eccentric exercises were applied only to the lower extremity muscles in the present study. Some studies have reported that when eccentric exercise was performed using both upper and lower extremity muscles, responses related to muscle injury appeared differently in both the upper and lower extremity muscles (Jamurtas et al., 2005; Saka et al., 2009). The effects of both the concentric and eccentric exercises applied in the present study on the upper extremity muscles (especially the elbow flexors) are still unknown. Lastly, the factors associated with muscle recovery in the study were limited to just maximal strength, muscle soreness, and CK. Since muscle recovery after eccentric exercise involves events such as inflammation, fibrosis, and/or myogenesis, future studies should include additional factors that can help observe such events to allow more diverse interpretation of the results.

\section{CONFLICT OF INTEREST}

No potential conflict of interest relevant to this article was reported. 


\section{REFERENCES}

Armstrong RB. Mechanisms of exercise-induced delayed onset muscular soreness: a brief review. Med Sci Sports Exerc 1984;16:529-538.

Brancaccio P, Maffulli N, Limongelli FM. Creatine kinase monitoring in sport medicine. Br Med Bull 2007;81-82:209-230.

Chen TC, Hsieh SS. Effects of a 7-day eccentric training period on muscle damage and inflammation. Med Sci Sports Exerc 2001;33:1732-1738.

Cheung K, Hume P, Maxwell L. Delayed onset muscle soreness: treatment strategies and performance factors. Sports Med 2003;33:145-164.

Donnelly AE, Clarkson PM, Maughan RJ. Exercise-induced muscle damage: effects of light exercise on damaged muscle. Eur J Appl Physiol Occup Physiol 1992;64:350-353.

Dupuy O, Douzi W, Theurot D, Bosquet L, Dugué B. An evidence-based approach for choosing post-exercise recovery techniques to reduce markers of muscle damage, soreness, fatigue, and inflammation: a systematic review with meta-analysis. Front Physiol 2018;9:403.

Howatson G, Van Someren K, Hortobágyi T. Repeated bout effect after maximal eccentric exercise. Int J Sports Med 2007;28:557-563.

Hyldahl RD, Chen TC, Nosaka K. Mechanisms and mediators of the skeletal muscle repeated bout effect. Exerc Sport Sci Rev 2017;45:24-33.

Jamurtas AZ, Theocharis V, Tofas T, Tsiokanos A, Yfanti C, Paschalis V, Koutedakis Y, Nosaka K. Comparison between leg and arm eccentric exercises of the same relative intensity on indices of muscle damage. Eur J Appl Physiol 2005;95:179-185.

Järvinen TA, Järvinen M, Kalimo H. Regeneration of injured skeletal muscle after the injury. Muscles Ligaments Tendons J 2014;3:337-345.

Kim J, Lee J. The relationship of creatine kinase variability with body composition and muscle damage markers following eccentric muscle contractions. J Exerc Nutrition Biochem 2015;19:123-129.

Koltyn KF. Analgesia following exercise: a review. Sports Med 2000;29:8598.

Lavender AP, Nosaka K. Changes in fluctuation of isometric force following eccentric and concentric exercise of the elbow flexors. Eur J Appl Physiol 2006;96:235-240.

Lund H, Vestergaard-Poulsen P, Kanstrup IL, Sejrsen P. Isokinetic eccentric exercise as a model to induce and reproduce pathophysiological alterations related to delayed onset muscle soreness. Scand J Med Sci
Sports 1998;8:208-215.

Manfredi TG, Fielding RA, O'Reilly KP, Meredith CN, Lee HY, Evans WJ. Plasma creatine kinase activity and exercise-induced muscle damage in older men. Med Sci Sports Exerc 1991;23:1028-1034.

Margaritis I, Tessier F, Verdera F, Bermon S, Marconnet P. Muscle enzyme release does not predict muscle function impairment after triathlon. J Sports Med Phys Fitness 1999;39:133-139.

McHugh MP. Recent advances in the understanding of the repeated bout effect: the protective effect against muscle damage from a single bout of eccentric exercise. Scand J Med Sci Sports 2003;13:88-97.

Nosaka K, Newton M. Repeated eccentric exercise bouts do not exacerbate muscle damage and repair. J Strength Cond Res 2002;16:117-122.

Proske U, Morgan DL. Muscle damage from eccentric exercise: mechanism, mechanical signs, adaptation and clinical applications. J Physiol 2001;537(Pt 2):333-345.

Saka T, Akova B, Yazici Z, Sekir U, Gür H, Ozarda Y. Difference in the magnitude of muscle damage between elbow flexors and knee extensors eccentric exercises. J Sports Sci Med 2009;8:107-115.

Saxton JM, Donnelly AE. Light concentric exercise during recovery from exercise-induced muscle damage. Int J Sports Med 1995;16:347-351.

Sayers SP, Clarkson PM, Rouzier PA, Kamen G. Adverse events associated with eccentric exercise protocols: six case studies. Med Sci Sports Exerc 1999;31:1697-1702.

Smith LL, Fulmer MG, Holbert D, McCammon MR, Houmard JA, Frazer DD, Nsien E, Israel RG. The impact of a repeated bout of eccentric exercise on muscular strength, muscle soreness and creatine kinase. Br J Sports Med 1994;28:267-271.

Sorichter S, Koller A, Haid C, Wicke K, Judmaier W, Werner P, Raas E. Light concentric exercise and heavy eccentric muscle loading: effects on CK, MRI and markers of inflammation. Int J Sports Med 1995;16: 288-292.

Sousa M, Teixeira VH, Soares J. Dietary strategies to recover from exercise-induced muscle damage. Int J Food Sci Nutr 2014;65:151-163.

Zainuddin Z, Sacco P, Newton M, Nosaka K. Light concentric exercise has a temporarily analgesic effect on delayed-onset muscle soreness, but no effect on recovery from eccentric exercise. Appl Physiol Nutr Metab 2006;31:126-134. 